\title{
THE
}

2-1-1994

\section{Boundary Effects and Spin Waves in Spin-Polarized Quantum Gases}

\author{
A. E. Meyerovich \\ University of Rhode Island, sfo101@uri.edu \\ S. Stepaniants \\ University of Rhode Island
}

Follow this and additional works at: https://digitalcommons.uri.edu/phys_facpubs

Terms of Use

All rights reserved under copyright.

\section{Citation/Publisher Attribution}

Meyerovich, A. E., \& Stepaniants, S. (1994). Boundary Effects and Spin Waves in Spin-Polarized Quantum Gases. Phys.Rev. B, 49(5), 3400-3408. doi: 10.1103/PhysRevB.49.3400

Available at: http://dx.doi.org/10.1103/PhysRevB.49.3400

This Article is brought to you for free and open access by the Physics at DigitalCommons@URI. It has been accepted for inclusion in Physics Faculty Publications by an authorized administrator of DigitalCommons@URI. For more information, please contact digitalcommons-group@uri.edu. 


\title{
Boundary effects and spin waves in spin-polarized quantum gases
}

\author{
A. E. Meyerovich and S. Stepaniants \\ Department of Physics, University of Rhode Island, Kingston, Rhode Island 02881
}

(Received 28 June 1993)

\begin{abstract}
Boundary conditions are derived for spin dynamics of spin-polarized quantum gases near nonmagnetic walls. We are interested mostly in boundary-induced line shifts and attenuation of spin waves, and in the possibility of having a macroscopic boundary condition for systems close to a Knudsen ballistic regime. We consider the effects caused by roughness of the wall and by surface adsorption. By a proper coordinate transformation, we reduce the problem of particle collisions with an inhomogeneous nonmagnetic wall to an equivalent problem with a specular homogeneous wall but with stochastic bulk imperfections. As a result, the boundary effects are described by some additional bulklike transverse spin-diffusion coefficient inversely proportional to the angular harmonics of the correlation function of surface inhomogeneities. This leads to an effective macroscopiclike boundary condition for transverse spin dynamics responsible for the boundary effects in spin-wave resonances. The situation changes drastically at low temperatures because of an appearance of an adsorbed boundary layer which renormalizes the molecular field near the wall, and leads to additional effective spin-exchange processes. The experimental implications for helium and hydrogen systems are discussed.
\end{abstract}

\section{INTRODUCTION}

Most of the interesting quantum phenomena in spinpolarized quantum systems, such as quantum gases and liquids, manifest themselves in spin dynamics. Spin dynamics of dilute spin-polarized quantum gases $\left(\mathrm{H} \downarrow,{ }^{3} \mathrm{He} \uparrow\right.$ component in dilute ${ }^{3} \mathrm{He} \uparrow-{ }^{4} \mathrm{He}$ liquid mixtures, etc.) is studied mainly (see Ref. 1 and references therein) at (ultra-) low temperatures when the mean free paths of particles may become quite long, making the boundary scattering as or even more important than the bulk collisions. ${ }^{2}$ Even an ultra-Knudsen ballistic regime seems to be accessible for modern low-temperature NMR techniques. This boundary scattering results in some dephasing and attenuation leading to a noticeable shift and broadening of spin-wave resonances or to a corresponding change in a spin-echo signal.

The boundary scattering is usually described in terms of effective cross section of diffusive scattering of particles by the wall. This description is not entirely appropriate for spin dynamics which corresponds to a dynamics of transverse (or off-diagonal) spin components of a density matrix and not to pure quantum states of particles.

One of the most paradoxical and useful features of transverse spin dynamics in dilute polarized quantum gases is the fact that the spin dynamics equations may be written ${ }^{1}$ in a "hydrodynamic" form (similar to the Leggett equations for dense Fermi liquids ${ }^{3}$ ) at arbitrary temperatures and arbitrary values $\delta \omega \tau$ and $k l\left(\delta \omega=\omega-\Omega_{0}\right.$, $\Omega_{0}=2 \beta H$ is the bare Larmor frequency, $\tau$, or more precisely, $\tau_{\perp}$, is the transverse spin relaxation time, and $l$ is the particle mean free path). The conditions of applicability of macroscopic hydrodynamiclike equations in the bulk are not $\delta \omega \tau, k l \ll 1$, but $\delta \omega, k v \ll\left|\Omega_{i}+i / \tau_{\perp}\right|$ where $v$ is the characteristic velocity of particles (Fermi velocity $v_{F}$ for degenerate fermions, or the thermal velocity $v_{T}$ at higher temperatures), and the internal frequency $\Omega_{i}$ characterizes the precession of the magnetic moment in the molecular field associated with the particle interaction. In case of large internal frequencies $\Omega_{i}$, the equations of spin dynamics can keep a "hydrodynamic" form at arbitrary values of $\delta \omega \tau_{\perp}$ and $k l$ (another similar example is propagation of waves in plasma in a collisionless regime where the role of a large internal frequency is played by the plasma frequency). In dilute quantum gases, the main term in $\Omega_{i}$ is $\Omega_{i}=-(4 \pi a \hbar / m)\left(N_{\uparrow}-N_{\downarrow}\right)$, where $a$ is the $s$-wave scattering length, $m$ is the (effective) mass of particles, and $N_{\uparrow, \downarrow}$ are the densities of spin-up and spin-down particles. The applicability of the theory ${ }^{1}$ of spin-polarized quantum gases is limited to relatively low (but not necessarily ultralow) temperatures and low densities $N=N_{\uparrow}+N_{\downarrow}$ when $v a^{2} N \ll \Omega_{i}$; in most of the cases this inequality requires large values of $\Omega_{i} \tau_{1} \gg 1$.

However, all the advantages of having macroscopic equations of spin dynamics in the bulk would be meaningless if one would not be able to supplement these equations with a macroscopic boundary condition. This problem becomes even more important close to a ballistic regime when the boundary scattering assumes the dominant role. Recently this problem has been approached by one of the authors ${ }^{4}$ from a phenomenological point of view. Though such an analysis shows possible forms of boundary conditions and demonstrates certain limitations of macroscopic equations, it is, of course, insufficient if one wants to describe the macroscopic boundary condition in terms of microscopic characteristics of the wall and individual scattering processes.

In this paper we study the effects of particles' scattering by nonmagnetic walls on spin dynamics and spin waves in spin-polarized quantum gases. In most of the cases we assume that the wall itself does not have any magnetic properties and does not contain particles with magnetic moments. Then the only two sources of boundary effects in spin dynamics are the scattering by surface 
inhomogeneities and the exchange processes with particles adsorbed by the wall. The latter effect becomes increasingly important at low temperatures if, as in the case of atomic hydrogen, the particles are attracted to and have a bound state on the walls. Then the densities of adsorbed layers increase exponentially with decreasing temperature. Both effects lead to an effective (spin) diffusion along the wall.

We want to demonstrate that such boundary effects can often be described adequately by a general "hydrodynamic" boundary condition,

$$
M+\Lambda n_{i} \nabla_{i} M=0,
$$

even if the mean free path is very long and the particles are nearly ballistic. Here $M$ is the magnetic moment per unit volume, $\mathbf{n}$ is the unit vector normal to the boundary (into the cell), and $\Lambda$ is the coefficient with the dimensionality of length. When Eq. (1) is valid, this coefficient should contain all the information about the boundary scattering.

In case of ideal specular walls, the coefficient $\Lambda \rightarrow \infty$. The scattering by surface inhomogeneities and the exchange with adsorbed particles lead to an effective spin diffusion along the wall, and, therefore, change the value of $\Lambda$. Below we solve the transport equation with the microscopic boundary conditions corresponding to both these types of wall scattering, and reduce the result to the macroscopic form (1). For some preliminary results of this work see Refs. 5.

\section{SCATTERING BY A ROUGH WALL}

The first problem is the scattering of particles by an inhomogeneous nonmagnetic wall. Collisions with a nonmagnetic wall conserve the magnetic moments of particles. Therefore, the only nontrivial effect of the boundary scattering is an additional two-dimensional (2D) spin diffusion of the particles along the wall which is caused by a randomness of scattering angles and reflects a random character of inhomogeneities of the wall.

The standard way to describe collisions with rough walls is to write a microscopic boundary condition for the transport equation as it is routinely done in the theory of metals (see, e.g., Ref. 6). The resulting integrodifferential equations are even more complicated in our case of mixed quantum states. We have adopted another very general method which can be used in a variety of situations. We performed a coordinate transformation which transforms an inhomogeneous wall into a smooth wall. This transformation is nonlinear and leads to additional random terms in the bulk Hamiltonian which reflect the inhomogeneities of the wall. We treat these random bulk perturbations of the Hamiltonian as some bulk imperfections which are described by a bulk collision integral in the transport equation. As a result, we reduce the transport problem of scattering by an inhomogeneous wall to an equivalent transport problem in a cell with specular homogeneous walls but with some stochastic bulk imperfections. By solving this transport problem, we obtain the additional (spin) diffusion coefficient which contains all the information on the boundary scattering relevant to spin dynamics.

We are interested in a transport equation for the momentum distribution of the transverse (to the magnetic field) components of the magnetic moment of the gas, $m\left(p_{x}, p_{\perp}, \varphi\right)=\operatorname{Tr}_{\sigma} \hat{\sigma}^{+} \hat{n}_{\sigma}(\mathbf{p})$, where $\hat{n}_{\sigma}(\mathbf{p})$ is the singleparticle density matrix, $p_{x}$ and $p_{\perp}$ are the components of the momentum $p$ perpendicular and parallel to the wall which has an average profile $x=$ const.

We consider a layer of a polarized quantum gas restricted by one rough wall $x=L-\xi(\mathrm{s})$ (s are the 2D coordinates $y, z$ in the plane of the wall), and a perfectly smooth uniform wall $x=0$. The function $\xi(\mathrm{s})$ is random, $\langle\xi(s)\rangle=0$, and all the observables should be averaged over $\xi$. The binary correlation function $\xi^{(2)}\left(\left|\mathbf{s}_{1}-\mathbf{s}_{2}\right|\right)$ $=\left\langle\xi\left(s_{1}\right) \xi\left(s_{2}\right)\right\rangle$ depends only on the distance $\left|s_{1}-s_{2}\right|$. The reflection from the second, perfect wall, is specular.

The shift of inhomogeneity from the boundary to the bulk is achieved by a coordinate transformation:

$$
X^{\prime}=\frac{x L}{L-\xi(\mathrm{s})} ; \quad Y^{\prime}=y ; Z^{\prime}=z .
$$

This transformation makes both walls, $X^{\prime}=0$ and $X^{\prime}=L$, smooth, and we can apply the simplest boundary condition $\Psi(0)=\Psi(L)=0$.

In principle, we can easily consider a layer with two rough surfaces, $x=\xi_{1}(\mathrm{~s})$ and $x=L-\xi_{2}(\mathrm{~s})$. Then the transformation

$$
X^{\prime}=\frac{x-\xi_{1}(\mathrm{~s})}{L-\xi_{1}(\mathrm{~s})-\xi_{2}(\mathrm{~s})} L ; \quad Y^{\prime}=y ; \quad Z^{\prime}=z
$$

will make both walls, $X^{\prime}=0$ and $X^{\prime}=L$, smooth. However, the only consequence will be that we will have to write, instead of the correlation function $\left\langle\xi(\mathbf{s}) \xi\left(\mathbf{s}^{\prime}\right)\right\rangle$, a sum of three correlation functions $\left\langle\xi_{1}(\mathbf{s}) \xi_{1}\left(\mathbf{s}^{\prime}\right)\right\rangle,\left\langle\xi_{2}(\mathbf{s}) \xi_{2}\left(\mathbf{s}^{\prime}\right)\right\rangle$, and $\left\langle\xi_{1}(\mathbf{s}) \xi_{2}\left(\mathbf{s}^{\prime}\right)\right\rangle$.

The coordinate transformation (2) changes the form of the bulk Hamiltonian. We can consider the effects of boundary scattering and bulk collisions of the particles independently from each other. Then, if one disregards the bulk collisions, the full Hamiltonian in new variables contains not only the quadratic part $\hat{H}_{0}$, but also an additional stochastic part ("perturbation") $\hat{V}$ which depends on $\xi$ :

$$
\begin{aligned}
& \hat{H}=\hat{H}_{0}+\hat{V}, \quad \hat{H}_{0}=\frac{p^{2}}{2 m}-\beta \hat{\sigma} H, \\
& \hat{V}=\frac{1}{2 m}\left(\frac{2 \xi}{L} p_{x}^{2}+x p_{x} \frac{\xi_{y}^{\prime}}{L} p_{y}+x p_{x} \frac{\xi_{z}^{\prime}}{L} p_{z}+\text { H.c. }\right] .
\end{aligned}
$$

We assume that the roughness $\xi$ is small, $\xi \ll L$, and keep only the first orders of $\xi$ and its derivatives. As a result, we have effectively transferred the surface imperfections into the bulk.

The random bulk imperfections $\hat{V}(3)$ should be included into the collision integral $L_{\text {coll }}$ for the Boltzmann equation using the perturbation theory: 


$$
\begin{gathered}
L_{\text {coll }}=\frac{p_{\perp}^{4}}{16 \pi L^{2} m} \int d \varphi \eta\left(p_{\perp}, \varphi-\phi\right)\left[m\left(p_{x}, p_{\perp}, \phi\right)-m\left(p_{x}, p_{\perp}, \varphi\right)\right] \\
\quad+\frac{p_{x}^{4}}{4 \pi L^{2} m} \int d \varphi \xi^{(2)}\left(p_{\perp}, \varphi-\phi\right)\left[m\left(p_{x}, p_{\perp}, \phi\right)-m\left(p_{x}, p_{\perp}, \varphi\right)\right], \\
\eta\left(p_{\perp}, \varphi-\phi\right) \equiv \xi^{(2)}\left(p_{\perp}, \varphi-\phi\right)[1-\cos (\varphi-\phi)]^{2} .
\end{gathered}
$$

Here $p_{x}$ and $\mathbf{p}_{1}$ are the components of momenta perpendicular and parallel to the wall, $\varphi$ and $\phi$, the angles between $p_{\perp}$, and some arbitrary axis for scattered and incident particles, respectively. The function $\xi^{(2)}\left(p_{\perp}, \varphi-\phi\right)$ is spatially uniform and depends only on the difference of the angles rather than on the angles themselves. The collision integral (4) corresponds to an elastic reflection in the direction perpendicular to the wall and to a random scattering in a $y z$ plane.

The collisions (4) with "bulk imperfections" (3) lead to an effective 2D spin diffusion in the plane of the wall and, therefore, affect the frequencies and the attenuation of spin waves. The collision integral (4) has the same form as for scattering by bulk impurities, and the calculations of the spin-diffusion coefficients are standard. The only nonzero components of the tensor of (spin) diffusion coefficients for transverse magnetic moment have the form

$D_{x x}=0, \quad D_{x y}=D_{y x}=D_{x z}=D_{z x}=0$

$D_{z z}=D_{y y}=\frac{8 \pi L^{2}}{\alpha m N} \int d p_{x} d^{2} p_{\perp} \frac{p_{\perp}^{2} M^{(0)}\left(p_{x}, p_{\perp}\right)}{p_{\perp}^{4}\left(\eta_{0}-\eta_{1}\right)+4 p_{x}^{4}\left(\xi_{0}-\xi_{1}\right)}$,

where $\eta_{k}\left(p_{\perp}\right)$ and $\xi_{k}\left(p_{\perp}\right)$ are the angular Fourier transforms of $\eta$ and $\xi^{(2)}$, respectively, $M^{(0)}(\mathbf{p})=n_{+}(\mathbf{p})$ $-n_{-}(\mathbf{p})$ is the equilibrium magnetic-moment distribution, $\alpha \equiv\left(N_{+}-N_{-}\right) / N$ is the degree of spin polarization, and $N_{ \pm}$are the numbers of spin-up and spin-down particles per unit volume. Equations (4) and (5) can be easily modified in order to accommodate the coordinate transformation (2a) instead of (2).

Equation (5) can be rewritten in a slightly different way:

$$
\begin{aligned}
D= & 4(2 \pi)^{4} \frac{\hbar}{m}\left[\frac{a L}{a^{*} R}\right]^{2} \frac{\hbar^{2}}{\alpha N a^{2}} \\
& \times \int \frac{d^{3} p}{(2 \pi \hbar)^{3}} \frac{a^{* 2} R^{2}}{\xi_{0}-\xi_{1}} \frac{p_{\perp}^{2} M^{(0)}\left(p_{x}, p_{\perp}\right)}{\zeta p_{\perp}^{4}+4 p_{x}^{4}}, \\
& \xi=\left(\eta_{0-} \eta_{1}\right) /\left(\xi_{0}-\xi_{1}\right),
\end{aligned}
$$

where $a$ is the atomic size, while $a^{* 2} \sim \xi^{(2)}(0)$ and $R$ characterize the height and the correlation radius of surface inhomogeneities. A simple estimate for this spindiffusion coefficient is

$$
D \sim \frac{\hbar}{m}\left(a^{3} N\right)^{-2 / 3}\left(\frac{a L}{a^{*} R}\right)^{2} \frac{T_{0}}{E},
$$

where the characteristic energy $E$ is the larger of $T$ and $T_{0}$ ( $T_{0}$ is the bulk degeneracy temperature). This should be compared with the bulk diffusion coefficient

$$
D_{v} \approx \frac{\hbar}{m}\left(a^{3} N\right)^{-2 / 3}\left(\frac{T_{0}}{E}\right)^{-1 / 2}\left(\frac{E}{T}\right)^{2} .
$$

Now we can supplement these boundary scattering results by taking into account the interaction of particles between themselves in the bulk. Using the Leggett equations for spin-polarized Fermi liquids and Eq. (5) for the transverse spin-diffusion coefficient, one can find imaginary terms in the spin-wave spectrum, and determine the surface-induced attenuation of spin waves (cf. Ref. 1). Then

$$
\begin{aligned}
& \omega(\mathbf{k})=\Omega_{0}+\frac{\gamma k^{2}}{3 \Omega_{i}}-\frac{i \gamma^{2} q^{2}}{9 \Omega_{i}^{2} D}, \mathbf{k}=\left(k_{x}, \mathbf{q}\right), \\
& \gamma(\alpha, T)=\frac{\left\langle v^{2}\right\rangle_{+} N_{+}-\left\langle v^{2}\right\rangle_{-} N_{-}}{N_{+}-N_{-}}, \\
& \left\langle v^{t}\right\rangle_{ \pm}=\frac{1}{N_{ \pm}} \int v^{t} n_{ \pm} d \Gamma, \quad \Omega_{i}=-\frac{4 \pi a \hbar}{m}\left(N_{+}-N_{-}\right),
\end{aligned}
$$

where $\left\langle v^{t}\right\rangle_{ \pm}(T)$ are the velocities of up and down spins averaged over the equilibrium distribution functions $n_{ \pm}$, and $a$ is the (bulk) $s$-wave scattering length. The equation (6) for the attenuation is applicable for quantum gases at all temperatures from the Boltzmann down to the degenerate region. In the low-temperature degenerate region

$\gamma=\frac{3}{5} \frac{v_{+}^{2} N_{+}-v_{-}^{2} N_{-}}{N_{+}-N_{-}}=\frac{3}{10} v_{F}^{2} \frac{(1+\alpha)^{5 / 3}-(1-\alpha)^{5 / 3}}{\alpha}$,

where $v_{ \pm}$are the Fermi velocities for up and down spins, and $v_{\mathrm{F}}$ is the Fermi velocity in the absence of polarization. In the Boltzmann region for quantum gases,

$$
\gamma=3 v_{T}^{2}, \quad v_{T}=(T / m)^{1 / 2}
$$

In the intermediate region, the function $\gamma(\alpha, T)$ can be parameterized as

$$
\gamma(\alpha, T)=v_{F}^{2} F_{1}\left(\alpha, \frac{T}{T_{0}}\right), \quad v_{F}=\frac{\left(3 \pi^{2} N\right)^{1 / 3} \hbar}{m},
$$

with the function $F_{1}(\alpha, t)$ given in Fig. 1.

Note, that the function $F_{1}\left(\alpha, T / T_{0}\right)$ and, therefore, $\gamma(\alpha, T)$, Eq. (6), practically do not depend on polarization $\alpha$. This is obvious at high temperatures when $\gamma$, Eq. (6b), is just a thermal velocity which for an ideal gas does not depend on polarization. However, even at low temperatures the polarization dependence of $\gamma$ is extremely weak. The largest dependence is at zero temperatures, Eq. (6a), when the function $F_{1}$ decreases gradually from 1 at zero polarization just to 0.95 at full polarization $\alpha=1$. The 
polarization dependence becomes even smaller with growing temperatures. Such an insignificant polarization dependence cannot even be seen on the scale of Fig. 1 (formally, the curve is plotted for $\alpha=0.9$ ). This peculiar lack of polarization dependence is rather important since the same function $\gamma(\alpha, T)$ determines the ratio of the transverse diffusion coefficient $D_{\perp}$ and the corresponding transverse relaxation time $\tau_{\perp}$ for dilute spin-polarized gases. ${ }^{1}$

Expression (5) for the transverse spin-diffusion coefficient shows how to write a macroscopiclike boundary condition for spin dynamics. A general macroscopic boundary condition at the wall $x=$ const should be linear in gradients and have the form (1). The results of boundary scattering and its importance are characterized by the value of the dimensionless parameter $k_{x} \Lambda$. As was demonstrated in Ref. 4, it is often possible to use Eq. (1), with $\Lambda$ expressed via $D(5)$, as a boundary condition for nonmagnetic walls.

In our geometry with one rough wall (finite $\Lambda$ ) at $x=0$ and a specular wall (infinite $\Lambda$ ) at $x=L$, the boundary condition (1) is equivalent to the eigenvalue equation

$$
\exp \left(2 i k_{x} L\right)=1-\frac{2}{k_{x} \Lambda-1} .
$$

As we will see, $\Lambda$ is real if one can neglect the bulk scattering, $\Omega_{i} \tau_{\perp} \gg 1$. The condition $k_{x} L=1$ determines the resonance surface adsorption. The eigenvalues (7) correspond to the frequency of the mode

$$
\omega(k)=\Omega_{0}+\frac{\gamma\left(k_{x}^{2}+q^{2}\right)}{3\left(\Omega_{i}+i / \tau_{\perp}\right)} .
$$

The comparison of Eqs. (7) and (8) with Eq. (6) gives the value of $\Lambda$.

The value of $k_{x} \Lambda$ depends on the magnitude of the parameter

$$
\sigma=\frac{E}{\hbar^{2} / m a^{2}} \frac{E}{\hbar\left(\Omega_{i}+i / \tau_{\perp}\right)} \frac{q^{2} a^{* 2}}{k_{x} L} .
$$

By the order of magnitude $\hbar \Omega_{i} \sim \alpha\left(N a^{3}\right)\left(\hbar^{2} / m a^{2}\right)$, $\hbar / \tau_{\perp} \sim\left(\mathrm{Na}^{3}\right)\left(E \hbar^{2} / m a^{2}\right)^{1 / 2}$. The spin-wave regime for dilute quantum gases corresponds to the condition $\Omega_{i} \tau_{1} \sim \alpha\left(\hbar^{2} / m a^{2} E\right)^{1 / 2} \gg 1$ if the polarization is not too low and the temperature is not too high. Then the parameter (9) is

$$
\sigma \approx\left(\frac{T}{T_{0}}\right)^{2} \frac{a N^{1 / 3}}{\alpha} \frac{\left(q a^{*}\right)^{2}}{k_{x} L}
$$

(the first factor should be disregarded at $T<T_{0}$ ). For very dilute gases $\mathrm{Na}^{3} \ll<$, and at not very high temperatures and low polarizations, the parameter $\sigma$ is usually small,

$$
\sigma<1 .
$$

This condition, which is always valid except for the case $q a^{*} \gg 1$ with anomalously large inhomogeneities, means that the effect of the wall is small.

Equation (7) has two such solutions corresponding to

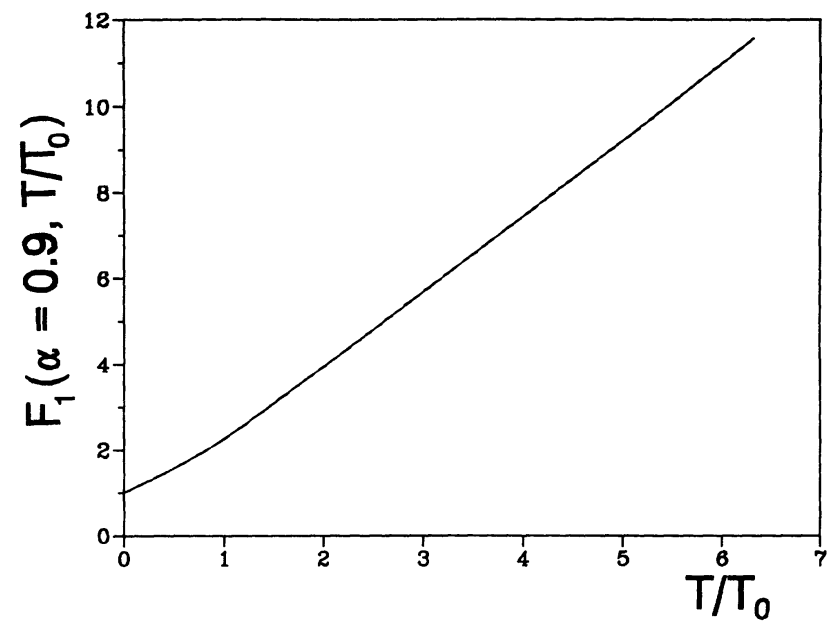

FIG. 1. Function $F_{1}\left(\alpha, T / T_{0}\right)$, Eq. (6). Formally, $\alpha=0.9$, but on this scale the polarization dependence is not noticeable.

$k_{x} \Lambda \ll<1$ and to $k_{x} \Lambda \gg 1$. In the former case, the comparison of Eqs. (6) $-(8)$ yields (cf. Ref. 4)

$$
\Lambda=\frac{\gamma q^{2} L}{12 k_{x}^{2}\left(\Omega_{i}+i / \tau_{\perp}\right) D}, \quad k_{x} \Lambda \sim \sigma \ll 1,
$$

meaning that the spin mode experiences a strong (diffusion) relaxation near the surface, and should have a node close to the wall. In the latter case, the characteristic length $\Lambda$ is large:

$$
\Lambda=\frac{12\left(\Omega_{i}+i / \tau_{\perp}\right) D}{\gamma q^{2} L}, k_{x} \Lambda \sim \frac{1}{\sigma} \gg 1,
$$

meaning a nearly zero current through the surface. This second case is close to specular refection in spin dynamics which corresponds to $\Lambda \rightarrow \infty$.

Equations (10) and (11) express the single parameter $\Lambda$ in the boundary condition (1) via the correlation function of surface inhomogeneities.

\section{BOUNDARY ADSORPTION AND SPIN DYNAMICS}

An adsorption of particles by the walls causes some major changes. Here one encounters two additional effects: effective exchanges of boundary particles between themselves, and exchanges between identical bulk and adsorbed particles. The former processes are suppressed when the surface mobility of adsorbed particles and their density are low, while the latter depend on the binding energy of adsorbed particles, and on the range of interaction between bulk and surface particles.

This additional exchange has a very strong effect on the boundary constant $\Lambda$ (1) if the density of adsorbed particles is large. This is always the case for spinpolarized atomic hydrogen at low temperatures. The hydrogen particles have a real bound state at the wall, and the density of adsorbed layers increases exponentially with decreasing temperature, $\rho=N R \exp \left(U_{0} / T\right)$. Such adsorbed layers have a very strong effect on $\Lambda$. However, for dense boundary layers the exchanges between bulk 
and surface particles are less important than the exchange or recombination of the surface particles. In this paper we neglect the interaction of the surface particles between themselves. Therefore our results are applicable to hydrogen systems at not very low temperatures and to ${ }^{3} \mathrm{He} \uparrow$ systems for which the boundary layers consist mostly of ${ }^{4} \mathrm{He}$ with a very small admixture of ${ }^{3} \mathrm{He}$ particles. Our results can be also used for ${ }^{3} \mathrm{He} \uparrow-{ }^{4} \mathrm{He}$ mixtures for a description of exchanges between bulk and surface states of ${ }^{3} \mathrm{He}$ at the free surface.

We are interested in the transport equation for a transverse (mixed) spin component of the single-particle density matrix of spin- $\frac{1}{2}$ particles with free and bound states. The exchange collisions between identical bulk and adsorbed particles are described by an appropriate component of the collision operator. Because of the exchange processes, this collision operator cannot be reduced to a simple standard combination of incoming and outgoing terms. In order to derive the collision integral, we started from a general transport equation for spin- $\frac{1}{2}$ particles $^{8}$ which we applied for delocalized free states (the density matrix $\rho_{\beta}^{\alpha}$ ) and the states with discrete energy levels which are localized on some traps (the density matrix $\widetilde{\rho}_{\beta}^{\alpha}$ ). Since we are dealing with a low-density system, the collisions of the free particles between themselves and with the trapped particles can be treated separately. In the latter case, the transport equation ${ }^{8}$ for the free particles has the form

$$
\begin{aligned}
-i \hbar \partial_{t} \rho_{\beta}^{\alpha}+[\hat{H}, \hat{\rho}]_{\beta}^{\alpha}= & -T_{\gamma \nu}^{\alpha \mu} \rho_{\beta}^{\gamma} \widetilde{\rho}_{\mu}^{v}+\rho_{\gamma}^{\alpha} \widetilde{\rho}_{\nu}^{\mu} T_{\beta \mu}^{+\gamma v} \\
& -T_{\gamma \nu}^{\alpha \mu} \rho{ }_{\lambda}^{\gamma} \widetilde{\rho}_{\chi}^{\nu} T_{\eta \delta}^{+\lambda \chi} G_{\beta \mu}^{+\eta \delta} \\
& +G_{\gamma \nu}^{\alpha \mu} T_{\lambda \chi}^{\gamma \nu} \rho_{\eta}^{\lambda} \widetilde{\rho}_{\delta}^{\chi} T_{\beta \mu}^{+\eta \delta}
\end{aligned}
$$

where the indices denote both spin and momentum variables, and $\hat{G}=\left(E-\hat{H}_{12}^{(0)}+i 0\right)^{-1}$ is the free two-particle propagator which is determined by the Hamiltonian $\hat{H}_{12}^{(0)}$ of noninteracting particles. The equation for the density matrix of the trapped particles $\widetilde{\rho}_{\beta}^{\alpha}$ is similar.

The $T$ matrix for scattering of delocalized particles on localized ones contains the term $t_{1}\left(\mathbf{p}, \mathbf{p}^{\prime}\right)$, which describes the direct scattering, and $t_{2}\left(p, p^{\prime}\right)$, which describes the scattering with a spin exchange,

$$
\begin{aligned}
T_{\mu \nu}^{\alpha \beta}\left(\mathbf{p}, \mathbf{p}^{\prime}\right)= & {\left[t_{1}\left(\mathbf{p}, \mathbf{p}^{\prime}\right)+\frac{1}{2} t_{2}\left(\mathbf{p}, \mathbf{p}^{\prime}\right)\right] \delta_{\mu}^{\alpha} \delta_{v}^{\beta} } \\
& +\frac{1}{2} t_{2}\left(\mathbf{p}, \mathbf{p}^{\prime}\right) \sigma_{\mu}^{\alpha} \sigma_{v}^{\beta}
\end{aligned}
$$

(here the indices stand only for the spin states). The (spin) exchanges $t_{2}\left(\mathbf{p}, \mathbf{p}^{\prime}\right)$ between the free multistate particles and identical trapped particles localized in some bound states near the walls can be described using the classical method of Ehrenfest and Oppenheimer. ${ }^{7}$ For a short-range interaction $V(\mathbf{r})$, the bulk-surface exchange integral $t_{2}$ is (see Appendix A):

$$
t_{2}\left(\mathbf{p}, \mathbf{p}^{\prime}\right) \approx V r_{0}^{3}\left(\frac{\lambda}{R}\right)^{2},
$$

where $R \sim a$ is the size of the bound state, $\lambda>a$ is the particle wavelength, and $r_{0}$ is the radius of interaction. In the opposite case of a long-range interaction, the exchange integral $t_{2}$ is equal to (see Appendix A)

$$
t_{2}\left(\mathrm{p}, \mathrm{p}^{\prime}\right) \approx V \lambda^{3} \frac{\lambda}{R} \frac{E}{U}
$$

where $U$ is the depth of the bound state and $E \ll U$ is the particle energy. The nonexchange part of the $T$ matrix, $t_{1}\left(\mathbf{p}, \mathbf{p}^{\prime}\right)$, describes the scattering by the trap with a particle; scattering by an empty trap (with or without trapping) should be introduced separately. However, it is quite obvious that the corresponding contribution to the collision integral will be of the same type as from $t_{1}\left(\mathbf{p}, \mathbf{p}^{\prime}\right)$; the only difference is a substitution of the number of trapped particles by the number of empty traps. Therefore, instead of introducing an additional scattering channel, we may work with a renormalized $t_{1}\left(\mathbf{p}, \mathbf{p}^{\prime}\right)$. Usually, $t_{1}$ is much larger than $t_{2}$.

The transport equation (12) has the mixed features of transport equations with particle-particle interactions and particle-impurity collisions. The major common feature with particle-particle collisions is the quantum identity of free and trapped particles. On the other hand, the immobility of trapped particles makes the process similar to the impurity scattering; this was taken into account by formally considering the effective mass of trapped particles to be very large, $m^{*} \rightarrow \infty$. The equations should also be averaged over random distribution of trapped particles along the wall. As a result of rather cumbersome transformations (see Appendix B), Eq. (12) for the mixed spin component of the density matrix and the similar equation of motion for the trapped particles are reduced to two coupled equations for bulk and surface transverse magnetizations, $\rho_{\downarrow \uparrow}$ and $\sigma_{\downarrow \uparrow}$. The collision operator for bulk particles is

$$
\begin{aligned}
\hbar L_{\mathrm{coll}}^{\mathrm{part}}= & 2 \pi e^{i k_{x} L} \sum_{p^{\prime}} \delta\left(\varepsilon^{\prime}-\varepsilon\right)\left\{\left(\left|t_{1}\right|^{2}+t_{1} t_{2}\right) N^{(s)}\left[\rho_{\downarrow \uparrow}\left(\mathbf{p}^{\prime}, L\right)-\rho_{\downarrow \uparrow}(\mathbf{p}, L)\right]+\left|t_{2}\right|^{2}\left[n\left(\mathbf{p}^{\prime}\right) \sigma_{\downarrow \uparrow}-N^{(s)} \rho_{\downarrow \uparrow}(\mathbf{p}, L)\right]\right\} \\
& +i e^{i k_{x} L} t_{2}\left[m_{z}(\mathbf{p}) \sigma_{\downarrow \uparrow}-\rho_{\downarrow \uparrow}(\mathbf{p}, L) M^{(s)}\right]
\end{aligned}
$$

where $n(\mathbf{p}), m_{z}(\mathbf{p}), N^{(s)}$, and $\boldsymbol{M}^{(s)}$ are the equilibrium densities of particles and magnetizations in the bulk and on the wall and $L$ is the coordinate of the wall. The collision operator for adsorbed particles is similar. The last, imaginary, term in (16) describes a change in the mean field near the wall. Other terms have a dissipative nature. The first square bracket in the right-hand side (rhs) of Eq. (16) corresponds to a simple difference of incoming and outgoing terms, while the second one has a purely exchange nature and reflects the quantum identity of free 
and trapped particles.

Collisions (16) with randomly distributed adsorbed particles result in an additional $2 \mathrm{D}$ spin diffusion in the plane of the wall with the diffusion coefficient

$$
\begin{aligned}
& D_{s}=\frac{a^{*} T / m}{i \alpha N^{(s)} t_{2}\left(p_{T}, \theta\right)+w}, \\
& w=\frac{N^{(s)} p_{T} m}{(2 \pi)^{2} \hbar^{3}} \int d \Omega\left[t_{2}^{2}+\left(t_{1}^{2}+t_{1} t_{2}\right)(1-\cos \theta)\right] .
\end{aligned}
$$

Here $p_{T}=(m T)^{1 / 2}, a^{*}$ is some parameter with the dimensionality of length which makes the dimensionality of $D_{s}$ the same as for a 3D diffusion coefficient (all observables contain only the ratio $a^{*} / D_{s}$, and not these parameters separately; $a *$ characterizes the "thickness" of the adsorbed layer). The imaginary part of $D_{s}$ comes from the molecular-field terms in the collision operator (16). This renormalization of the molecular-field near the walls also leads to some attenuation since it causes a dephasing of precessions in the bulk and near the wall.

This 2D diffusion enters the equation for the spin current $\mathbf{J}_{i}$ (Ref. 4)

$$
\begin{aligned}
-\frac{i m}{T}\left[\Omega_{i}+\frac{i}{\tau_{\perp}}\right] \mathbf{J}_{i}+\frac{\partial M}{\partial x_{i}}=- & \frac{a^{*}}{D_{s}}[\delta(x)+\delta(x-L)] \\
& \times\left[\mathbf{J}_{i}-n_{i} n_{k} \mathbf{J}_{k}\right],
\end{aligned}
$$

where $\mathbf{n}$ is the unit vector perpendicular to the walls at $x=0$ and $x=L$. According to Ref. 4, the $\delta$-like boundary condition (18) is equivalent, in nearly specular conditions $k_{x} \Lambda \gg 1$, to the macroscopic boundary condition (1) with

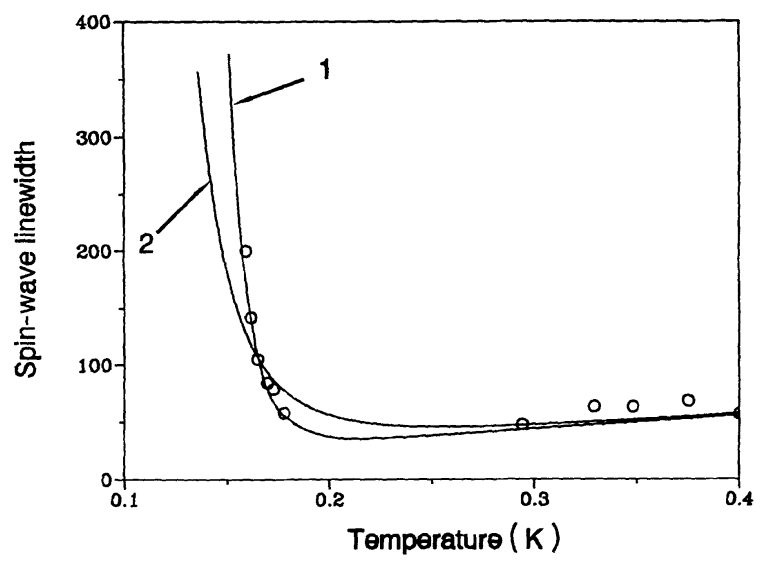

FIG. 2. Temperature dependence of the spin-wave linewidth. Experimental points and fit 1 are taken from Ref. 2, curve 2, Eq. (23).

$$
\Lambda=-\frac{D_{s}}{q^{2} a^{*}} \frac{m}{T}\left(\Omega_{i}+\frac{i}{\tau_{\perp}}\right)
$$

which is similar to Eq. (11). In an opposite case $k_{x} \Lambda \ll 1$ the expression for $\Lambda$ is similar to Eq. (10).

The transport equation (12) is valid only for the Boltzmann distribution of the bulk particles. These results can be easily generalized to lower temperatures. At arbitrary temperatures the derivation of an appropriate transport equation is much more cumbersome. We will not go here into the details of a diagrammatic derivation, ${ }^{9}$ and will give only the expression for the collision integral (without molecular-field terms) which should be used instead of Eq. (16):

$$
\begin{array}{r}
\hbar L_{\mathrm{coll}}^{\mathrm{part}}=\pi e^{i k_{x} L} \sum_{p^{\prime}} \delta\left(\varepsilon^{\prime}-\varepsilon\right)\left(\left[2\left|t_{1}\right|^{2}+t_{1}^{*} t_{2}+t_{1} t_{2}^{*}\right]\left[N^{(s)}-N_{\uparrow \uparrow}^{(s) 2}-N_{\downarrow \downarrow}^{(s) 2}\right]\left(\rho_{\downarrow \uparrow}^{\prime}-\rho_{\downarrow \uparrow}\right)\right. \\
+\left|t_{2}\right|^{2}\left\{\left[n \sigma_{\downarrow \uparrow}-\rho_{\downarrow \uparrow}\left(2-N^{(s)}\right)\right]\left(N^{(s)}-N_{\uparrow \uparrow}^{(s)} n_{\uparrow \uparrow}^{\prime}-N_{\downarrow}^{(s)} n_{\downarrow \downarrow}^{\prime}\right)\right. \\
\left.\left.-\left[N^{(s)} \rho_{\downarrow \uparrow}-\sigma_{\downarrow \uparrow}(2-n)\right]\left(n^{\prime}-N_{\uparrow \uparrow}^{(s)} n_{\uparrow \uparrow}^{\prime}-N_{\downarrow}^{(s)} n_{\downarrow \downarrow}^{\prime}\right)\right\}\right) .
\end{array}
$$

The consequent calculations are close to those above, and the final expression for $\Lambda$ differs from Eq. (19) by a substitution of $m / T$ by a function similar to $\gamma\left(\alpha, T / T_{0}\right)$, Eq. (6).

The above equations assume that the density of adsorbed particles is small, and the interaction between them is negligible. This should be true for ${ }^{3} \mathrm{He} \uparrow-{ }^{4} \mathrm{He}$ mixtures where the adsorbed layer at the cell walls consists mostly of ${ }^{4} \mathrm{He}$ with a very small admixture of ${ }^{3} \mathrm{He}$ atoms. With the lack of adequate low-temperature experiments for helium mixtures, we applied our results to spinpolarized atomic hydrogen for which the density of the adsorbed layer increases exponentially at low temperatures. Our results should provide a good description of wall contributions only at relatively high temperatures, when the density of the adsorbed layer is still low.

The effect of surface adsorption on the spectrum of spin waves has been measured by the Cornell group. ${ }^{2}$ In the presence of a magnetic-field gradient $G$ the spatial profile magnetic moment $M(x)$ is described by the Airy function

$$
\Phi\left[-\left[x-\frac{\omega}{\beta G}\right]\left[\frac{\beta G}{D_{v}} \frac{1+\Omega_{i}^{2} \tau_{\perp}^{2}}{i+\Omega_{i} \tau_{\perp}}\right]^{1 / 3}\right],
$$

where $\beta$ is the particle magnetic moment and $D_{v}$ is the bulk spin-diffusion coefficient. The boundary condition (1) together with the above value of the surface spindiffusion coefficient $D_{s}$ lead to the following eigenvalue equation for the spin dynamics [cf. Eq. (7)]:

$$
\begin{aligned}
& \Phi(x=0)+\Lambda \Phi_{x}^{\prime}(x=0)=0, \\
& \Lambda=-i \frac{\left(\Omega_{i}+i / \tau_{\perp}\right)}{q^{2} w},
\end{aligned}
$$

where $q$ is the wave vector along the wall. The solution of this equation gives the following value for the imaginary part of the spectrum:

$$
\omega^{\prime \prime}=\frac{q^{2} w \beta G}{\Omega}\left(\frac{T}{m \beta G \Omega}\right)^{2 / 3}+\frac{(\gamma G)^{2 / 3}}{3 \Omega^{4 / 3}} n a^{2}\left(\frac{T}{m}\right)^{5 / 6} .
$$


The first term in this equation describes the attenuation caused by the wall, while the second term is due to the bulk interactions. The function (23) is plotted in Fig. 2.

The agreement with experimental data is quite good at relatively high temperatures where the density of the adsorbed layer is small. At lower temperatures the density of adsorbed hydrogen atoms increases exponentially, and the experimental data deviate from the theoretical curve confirming the importance of interaction of adsorbed particles at high coverage [at low temperatures the index of the exponent in $\omega^{\prime \prime}(T)$ is twice the index in the density of adsorbed particles $N^{(s)}(T)$ ].

\section{SUMMARY}

We analyzed two boundary effects in spin dynamics of spin-polarized quantum gases: scattering by surface inhomogeneities and exchange with adsorbed particles. Both processes lead to an additional spin diffusion in the plane of the wall. The corresponding spin-diffusion coefficients are expressed via the characteristics of the wall and the density of adsorbed particles. We derived an effective boundary condition which covers these two surface processes. The results are important at low temperatures when the particle mean free paths are very long. The main experimental application of the work concerns spin-dynamics experiments in ${ }^{3} \mathrm{He} \uparrow-{ }^{4} \mathrm{He}$ mixtures at ultralow temperatures. The direct application to hydrogen systems at low temperatures is still limited to the extent to which the interaction and recombination of adsorbed hydrogen atoms are negligible.

In the future we plan to include the interaction between the adsorbed particles and some form of a magnetic interaction of the bulk and adsorbed particles with the particles of the wall.

\section{ACKNOWLEDGMENTS}

We would like to thank K. Musaelian for numerous valuable suggestions. One of the authors (A.M.) is grateful to G. Frossati and his group at Kamerlingh Onnes Laboratory for hospitality and useful discussions. The work has been supported by NSF Grant No. DMR 9100197 and NATO Grant No. CRG 910283.

\section{APPENDIX A}

The probability of particle exchange during the scattering of a free particle on a trapped one is given by the matrix element

$t_{2}=\int \Psi_{k^{\prime}}^{*}\left(r^{\prime}\right) \Phi^{*}\left(\mathbf{r}^{\prime}\right) V\left(\mathbf{r}-\mathbf{r}^{\prime}\right) \Psi_{k}(\mathbf{r}) \Phi(\mathbf{r}) d^{3} r d^{3} r^{\prime}$,

where $V(\mathbf{r})$ is the particle interaction, $\Psi(\mathbf{r})$ and $\Phi(\mathbf{r})$ are the wave functions of free and trapped particles, respectively. For a spherically symmetrical, deep and narrow single-level trap, the integral (A1) can be rewritten as

$$
\begin{array}{rl}
t_{2} \approx \int_{0}^{R} \int_{0}^{R} & V\left(\left|\mathbf{r}-\mathbf{r}^{\prime}\right|\right) \frac{\sin k r^{\prime}}{k_{1} r} \Phi^{*}\left(r^{\prime}\right) \\
& \times \frac{\sin k r}{k_{1} r} \Phi(r) d^{3} r d^{3} r^{\prime}
\end{array}
$$

where $R$ is the radius of the trap, $k=[2 m(E+U)]^{1 / 2} / \hbar$ and $k_{1}=[2 m E]^{1 / 2} / \hbar$ are the wave vectors of the free particle with the energy $E$ inside and outside the trap of the depth $U, k R \gg 1$, and we have neglected all insignificant dimensionless constant factors of the order of 1 .

If the interaction between the particles has a very short range $r_{0} \ll R, V(\mathbf{r})=\left(V / r_{0}^{3}\right) \delta\left(\mathbf{r}-\mathbf{r}^{\prime}\right)$, and Eq. (A2) takes the form

$$
t_{2} \approx V r_{0}^{3} \int_{0}^{R}\left|\frac{\sin k r}{k_{1} r} \Phi(r)\right|^{2} r^{2} d r .
$$

Since $\sin k r$ is a rapidly oscillating function inside the trap while $\Phi(r) \sim 1 / R^{3 / 2}$ changes very slowly, $\sin ^{2} k r$ in the integrand can be substituted by $\frac{1}{2}$. Then a simple estimate of the integral (A3) yields

$$
t_{2} \approx V r_{0}^{3}\left[\frac{\lambda}{R}\right)^{2}
$$

where $\lambda \sim 1 / k_{1}$ is the free particles' wavelength.

In the opposite case of a long-range interaction, $V\left(\left|\mathbf{r}-\mathbf{r}^{\prime}\right|\right)=V_{0}$, the integral (A2) reduces to

$$
t_{2} \approx V_{0}\left|\int_{0}^{R} \frac{\sin k r}{k_{1} r} \Phi(r) r^{2} d r\right|^{2} .
$$

Now we have a rapidly oscillating integrand with a zero average which leads to a following estimate for $t_{2}$ :

$$
t_{2} \approx V_{0} \lambda^{3} \frac{\lambda}{R} \frac{E}{U}
$$

\section{APPENDIX B}

We are interested in a transport equation for particles which can be in free delocalized states or be trapped by some randomly distributed deep and narrow traps. The transport equation for a single-particle density matrix $\rho_{\beta}^{\alpha}$ of particles with internal states (in our case, spin states) has the following form: ${ }^{8}$

$$
\begin{aligned}
\partial_{t} \rho_{\beta}^{\alpha}+\frac{i}{\hbar}[H, \rho]_{\beta}^{\alpha}=\frac{i}{\hbar}( & -T_{\gamma \nu}^{\alpha \mu} \rho_{\beta \mu}^{\gamma v}+\rho_{\gamma \nu}^{\alpha \mu} T_{\beta \mu}^{+\gamma \nu} \\
& -T_{\gamma \nu}^{\alpha \mu} \rho_{\lambda k}^{\gamma v} T_{\eta \delta}^{+\lambda k} G_{\beta \mu}^{+\eta \delta} \\
& \left.+G_{\gamma \nu}^{\alpha \mu} T_{\lambda k}^{\gamma v} \rho_{\eta \delta}^{\lambda k} T_{\beta \mu}^{+\eta \delta}\right),
\end{aligned}
$$

where $\rho_{\mu \nu}^{\alpha \beta}$ is the two-particle density matrix, $\hat{T}$ is the scattering $T$ matrix, $G$ is the Green's function, Greek indices include both momentum and spin states, and we still do not separate free and localized particles. Here and below we assume summation over redundant indices.

Since we are dealing with a low-density system, all interaction processes can be treated separately, independently from each other. Here we are interested only in (exchange) interaction between free and trapped particles. Therefore, it is sufficient to write the two-particle density matrix $\rho_{\mu \nu}^{\alpha \beta}$ as a product of single-particle distributions in free and trapped states, and neglect the terms quadratic in respective densities. We do not have to symmetrize this product (or the two-particle density matrix) as has been done in Ref. 8 because the symmetrization is already 
implied by a proper form of the $T$ matrix, Eq. (13), which takes into account the exchanges between identical free and trapped particles. Thus we can formally consider trapped and free particles as distinguishable and represent the two-particle density matrix as a direct product of one-particle density matrices for trapped and free particles:

$$
\rho_{\mu \nu}^{\alpha \beta}=\rho_{\mu}^{\alpha} \widetilde{\rho}_{v}^{\beta}, \quad \widetilde{\rho}_{j}^{i}\left(\mathbf{p}, \mathbf{p}^{\prime}\right)=\widetilde{\rho}_{j}^{i} \delta_{\mathbf{p}^{\prime}}^{\mathbf{p}},
$$

where $\rho$ and $\widetilde{\rho}$ are free-particle and trapped-particle density matrices, respectively, and the indices $(i, j)$ denote only the spin states. The momentum $\delta$-function in the density matrix for the trapped particles corresponds to a uniform spatial distribution of traps. With the help of Eq. (B2), Eq. (B1) reduces to Eq. (12).

In these notations, Eq. (B1) is the transport equation for free particles. This equation should be supplemented (see below) by an analogous equation for trapped particles. At this stage it is enough to substitute the operator $\rho$ by $\tilde{\rho}$ in the left-hand side (lhs) of Eq. (B1).
The $T$ matrix and the Green's function in Eq. (B1) contain the $\delta$-functions which correspond to the momentum and energy conservation laws for two colliding particles. The conservation laws could be simplified since the trapped particles have no momenta, while their energy (or, more precisely, the part which is not associated with spin states) remains constant throughout the collisions. This can be taken into account by assuming very large effective masses, $m^{*} \rightarrow \infty$, for trapped particles and disregarding their momenta in the arguments of the $\delta$ functions which reflect the momentum conservation law. This procedure leads to a substantial simplification of momentum dependence of Eq. (B1), but does not affect the spin variables. The spin indices in exchange systems are always decoupled from the momentum variables and can be taken care of separately.

We will illustrate this procedure on the example of the third term in the rhs of Eq. (B1). After substitution of the energy and momentum $\delta$ functions which are contained in the $T$ matrix, this term, $L_{3}$, assumes the following form:

$$
\begin{aligned}
& L_{3}=-T_{\alpha_{2} \mathrm{p}_{2}, \alpha_{3} \mathrm{p}_{3}}^{\alpha \mathrm{p}, \alpha_{1} \mathrm{p}_{1}} \rho_{\alpha_{4} \mathrm{p}_{4}, \alpha_{5} \mathrm{p}_{5}}^{\alpha_{2} \mathrm{p}_{2}, \alpha_{3} \mathrm{p}_{3}} T_{\alpha_{6} \mathrm{p}_{6}, \alpha_{7} \mathrm{p}_{7}}^{+\alpha_{4} \mathrm{p}_{4}, \alpha_{5} \mathrm{p}_{5}} G_{\alpha^{\prime} \mathrm{p}^{\prime}, \alpha_{1} \mathrm{p}_{1}}^{+\alpha_{6} \mathrm{p}_{6}, \alpha_{7} \mathrm{p}_{7}} \\
& =-T_{\alpha_{2} \alpha_{3}}^{\alpha \alpha_{1}}\left(\mathbf{p}-\mathbf{p}_{1}, \mathbf{p}_{2}-\mathbf{p}_{3}\right) \rho_{\alpha_{4}}^{\alpha_{2}}\left(\mathbf{p}_{2}, \mathbf{p}_{4}\right) \tilde{\rho}_{\alpha_{5}}^{\alpha_{3}} \delta\left(\mathbf{p}_{3}-\mathbf{p}_{5}\right) T_{\alpha^{\prime} \alpha_{1}}^{+\alpha_{4} \alpha_{5}}\left(\mathbf{p}_{4}-\mathbf{p}_{5}, \mathbf{p}_{6}-\mathbf{p}_{7}\right) \\
& \times \delta\left(p+p_{1}-p_{2}-p_{3}\right) \delta\left(p_{4}+p_{5}-p_{6}-p_{7}\right) \delta\left(p_{6}-p^{\prime}\right) \delta\left(p_{7}-p_{1}\right) \delta\left(\varepsilon_{4}+\varepsilon_{5}-\varepsilon^{\prime}-\varepsilon_{1}\right) \\
& =-T_{\alpha_{2} \alpha_{3}}^{\alpha \alpha_{1}}\left(\mathbf{p}-\mathbf{p}_{1}, 2 \mathbf{p}_{2}-\mathbf{p}-\mathbf{p}_{1}\right) \rho_{\alpha_{4}}^{\alpha_{2}}\left(\mathbf{p}_{2}, \mathbf{p}^{\prime}+\mathbf{p}_{2}-\mathbf{p}\right) \tilde{\rho}_{\alpha_{5}}^{\alpha_{3}} \\
& \times T_{\alpha^{\prime} \alpha_{1}}^{+\alpha_{4} \alpha_{5}}\left(\mathbf{p}^{\prime}+2 \mathbf{p}_{1}-2 \mathbf{p}-\mathbf{p}_{1}, \mathbf{p}^{\prime}-\mathbf{p}_{1}\right) \delta\left[\frac{\left(\mathbf{p}^{\prime}+\mathbf{p}_{2}-\mathbf{p}\right)^{2}}{2 m}-\frac{p^{2}}{2 m}\right] .
\end{aligned}
$$

We should neglect energies $\varepsilon_{5}$ and $\varepsilon_{1}$ in the energy $\delta$ function since they correspond to trapped particles. We are interested only in the mixed spin component of the transport equation, i.e., in the case $\alpha=\uparrow, \alpha^{\prime}=\downarrow$. After substituting the $T$ matrix from Eq. (13) and performing both the Wigner transform and summation over redundant spin indices we get

$$
\begin{aligned}
\mathbf{W}\left(L_{3}\right)=-\pi i \delta\left(\varepsilon_{2}-\varepsilon\right)[ & \left|t_{1}\left(\mathbf{g}, \mathbf{g}^{\prime}\right)\right|^{2} \rho_{\downarrow}^{\uparrow}\left(\mathbf{p}_{2}, \mathbf{r}\right) N+t_{2}\left(\mathbf{g}, \mathbf{g}^{\prime}\right) t_{1}^{*}\left(\mathbf{g}, \mathbf{g}^{\prime}\right) \rho_{\downarrow}^{\mu}\left(\mathbf{p}_{2}, \mathbf{r}\right) \tilde{\boldsymbol{\rho}}_{\mu}^{\uparrow} \\
& \left.+t_{1}\left(\mathbf{g}, \mathbf{g}^{\prime}\right) t_{2}^{*}\left(\mathbf{g}^{\prime}, \mathbf{g}\right) \rho_{\mu}^{\uparrow}\left(\mathbf{p}_{2}, \mathbf{r}\right) \widetilde{\rho}_{\downarrow}^{\mu}+t_{2}\left(\mathbf{g}, \mathbf{g}^{\prime}\right) t_{2}^{*}\left(\mathbf{g}^{\prime}, \mathbf{g}\right) n\left(\mathbf{p}_{2}, \mathbf{r}\right) \tilde{\boldsymbol{\rho}}_{\downarrow}^{\uparrow}\right] \\
& \mathbf{g}=\mathbf{p}-\mathbf{p}_{1}, \quad \mathbf{g}^{\prime}=2 \mathbf{p}_{2}-\mathbf{p}-\mathbf{p}_{1} .
\end{aligned}
$$

Here W $\left(L_{3}\right)$ means the Wigner transform of $L_{3}, \mathbf{g}$, and $\mathbf{g}^{\prime}$ are the relative momenta of the particles before and after collision.

The fourth term in Eq. (B1) has nearly the same structure as the third one in Eqs. (B3) and (B4). The first two terms in Eq. (B1), which are linear in the $T$ matrix, are different. However, these terms describe the forward (zero angle) scattering and can be transformed into quadratic in $T$ terms [similar to (B3) and (B4)] with the help of the optical theorem

$$
T(\mathbf{p}, \mathbf{p})^{+}-T(\mathbf{p}, \mathbf{p})=2 \pi i T^{+} \delta\left(E-H^{0}(1,2)\right) T .
$$

Equation (B4) shows the structure of the collision operator in the case of spatially uniform distribution of traps. In our case the traps are uniformly distributed, but only in one specific plane. This means that the averaged single-particle density matrix for the trapped parti- cles, $\widetilde{\rho}$, in contrast to Eq. (B2), contains only a $2 \mathrm{D} \delta$ function of momenta (in the plane of the wall), while the third component should be substituted by the factor $\exp \left(i k_{x} L\right)$. As a result, the rhs of Eq. (B1) reduces to Eq. (16).

The transport equation for the trapped particles could be obtained from Eq. (12) by substituting the density matrices $\rho \leftrightarrow \widetilde{\rho}$. Then, in order to get rid of the $\delta$ function (B2) in the lhs of the transport equation, one should perform an additional integration over the momenta. As a result, the rhs of the equation for $\widetilde{\rho}_{\downarrow}^{\uparrow} \equiv \sigma_{\uparrow \downarrow}$ becomes essentially the same as the integrated Eq. (16):

$$
\begin{aligned}
\hbar L_{\text {coll }}^{\text {traart }}= & 2 \pi \sum_{p, p^{\prime}} \delta\left(\varepsilon^{\prime}-\varepsilon\right)\left|t_{2}\right|^{2} \\
& \times\left[N^{(s)} \rho_{\downarrow \uparrow}\left(\mathbf{p}^{\prime}, L\right)-n\left(\mathbf{p}^{\prime}\right) \sigma_{\downarrow \uparrow}\right] \\
& -i \sum_{\mathbf{p}} t_{2}\left[m_{z}(\mathbf{p}) \sigma_{\downarrow \uparrow}-\rho_{\downarrow \uparrow}(\mathbf{p}, L) M^{(s)}\right] .
\end{aligned}
$$


${ }^{1}$ A. E. Meyerovich, in Helium Three, edited by W. P. Halperin and L. P. Pitaevskii (North-Holland, Amsterdam, 1990), pp. 757-879.

${ }^{2}$ N. P. Bigelow, J. H. Freed, and D. M. Lee, Phys. Rev. Lett. 63, 1609 (1988).

${ }^{3}$ A. J. Leggett, J. Phys. C 3, 448 (1970).

${ }^{4}$ A. E. Meyerovich, J. Low Temp. Phys. 86, 311 (1992).

${ }^{5}$ S. Stepaniants and A. E. Meyerovich, J. Low Temp. Phys. 89, 539 (1992); Boundary Effects in Transverse Spin Dynamics of
Low Temperature Quantum Gases, Proceedings of the 20th International Conference on Low Temperature Physics (LT-20, Eugene, Oregon, 1993) [Physica B + C (to be published)].

${ }^{6}$ L. A. Falkovskii, Zh. Eksp. Teor. Fiz. 58, 1830 (1970) [Sov. Phys. JETP 31, 981 (1970)].

${ }^{7}$ P. Ehrenfest and J. R. Oppenheimer, Phys. Rev. 37, 333 (1931).

${ }^{8}$ A. E. Meyerovich, Phys. Rev. B 39, 9318 (1989).

${ }^{9}$ S. Stepaniants, K. A. Musaelian, and A. E. Meyerovich (unpublished). 